\title{
Vesical Hydatid Cyst: A Rare Case
}

Youssef Kerroum*, Oukli, Maliki, Khedid, kheddach, Hrichiou, Zentar

Hopital Militaire d'instruction mohammed V, Rabat, Morocco

*Corresponding author: Youssef Kerroum

Abstract

This observation is probably very rare. It helps to remember that the hydatid cyst spares no organ. Hydaturia is the pathognomonic clinical sign of a cystic rupture in the excretory tract. Ultrasound is the first-line screening examination. CT gives more precise information on the number, the topography and on certain morphological elements of the cyst such as calcifications.

Keywords: Vesical Hydatid Cyst, Rare Case, Hydaturia, Ultrasound.

Copyright @ 2020: This is an open-access article distributed under the terms of the Creative Commons Attribution license which permits unrestricted use, distribution, and reproduction in any medium for non-commercial use (NonCommercial, or CC-BY-NC) provided the original author and source are credited.

\section{INTRODUCTION}

Hydatidosis is endemic in Morocco, a disease caused by the accidental ingestion of Echinococcus granulosus eggs. Pulmonary and hepatic sites are the most frequent. The retrovesical location of the hydatid cyst is rare, if not exceptional, and often misleading, accounting for less than $4 \%$ of all visceral sites [1]. The kidney is the most common localization of the urogenital tract.

Our case reminds surgeons of the importance of always calling first a hydatid cyst before any other tumoral mass of the cystic wall, in an endemic country and being alarmed at the risk of surgical contamination.

\section{MEDICAL OBSERVATION}

The case is about a 61 - year - old patient, followed for viral hepatitis $\mathrm{HCV}$, a pelvic mass was accidentally discovered during a check-up of his hepatitis. On the clinical side, the patient was asymptomatic without any notion of alteration of the general state.

The patient underwent an ultrasound that only objectified a renal cyst, for which he subsequently benefited from a Urinary tract CT scan (figure 1), which showed 2 cortical renal cysts partitioned, one on the right and the other on the left, and an intraperitoneal lateral-vesical tissue process, suggesting a stromal tumor in the first place. Given the doubt of diagnosis, a pelvic MRI was made (figure 2), which was in favor of a fibrous tumor. The decision to operate the patient was made by the staff of the department; a surgical exploration found a hydatid cyst at the expense of the bladder wall (figure 3), the diagnostic of a vesical hydatid cyst type 3 of the Gharbi classification had been retained [2].

The patient has benefited from a resection of the protruding dome, and an aspiration of the contents of the cyst, along with a serum and hydrogen peroxide wash.

The pathological anatomy has confirmed the Diagnistoc retained afterward.

\section{DISCUSSION}

In the literature, 20 cases of retrovesical localizations have been recorded during the last 15 years, with a male predominance (2.5 men for 1 woman) [3] the hydatid localization of the bladder wall is exceptional [4].

The accidental finding of a hypogastric or bladder-swelling in an adult must, in a zone of hydatic endemic, suggest the retro-vesical cyst, especially since sometimes the cyst is opened in the bladder with haematuria and hydatids [5]. In general, UIV and ultrasound make the diagnosis of localization easy.

The Clinical manifestations include pelvic mass, flank pain, or acute urine retention. Urinal Hydatidosis is the only clinical sign characteristic of the disease, it indicates the opening of the cyst in the excretory pathways 
Often urogenital hydatidosis is secondary to another visceral involvement [6], most often hepatic and ultrasound is the screening test of choice. It allows exploring the entire abdominopelvic cavity. It allows the diagnosis of a bladder hydatidosis and essentially the analysis of intracystic structures (partitions, daughter vesicles and hydatid sand). The limitations of ultrasound are the difficulty in counting all the hydatid localizations of the abdominopelvic cavity and to establish the starting point of the cyst especially when it is voluminous.

CT scan is the exam of preference in the exploration of hydatid disease. It is very sensitive for the detection of calcifications, of the daughter vesicles and better defines the relations with the neighborhood organs and the precise count of the cysts. In our observation, it made it possible to discover the mass and also allowed the exploration of the entire peritoneal cavity in search of other sites.

The Gharbi classification presents the different aspects of hydatid cysts according to their evolutionary stage [2]. It distinguishes several types of cysts.

In our case it is in favor of type 4 with accumulation of hydatidosis sand, hydatids compressed and calcifications which can lead to the pseudo-tumor form
Magnetic resonance imaging is not a first-line technique in hydatid disease. It is justified only when the other sectional images do not make it possible to establish a definite diagnosis. Images of hydatid vesicles result in a hyposignal on the T1-weighted sequences and a hypersignal on the T2-weighted sequences. The wall of the lesion in hypointense signal in $\mathrm{T} 1$ and in $\mathrm{T} 2$ is raised discreetly after injection of Gadolinium [7].

This case is probably very rare. It reminds us that the hydatid cyst spares no organ

\section{CONCLUSION}

In an endemic country of hytatidosis, the diagnosis of hydatid cyst with a mass should not be ruled out. It can be asymptomatic as it can manifest itself by a hydatury that is pathognomonic. Ultrasound is the first-line screening test. The CT provides more precise information on the number, topography and on some morphological elements of the cyst such as calcifications. Treatment is surgical: kystoperikystectomy, with the importance of the protection of the surgical field to avoid a migration of the parasite, considering the important risk of contamination. The evolution is often favorable.

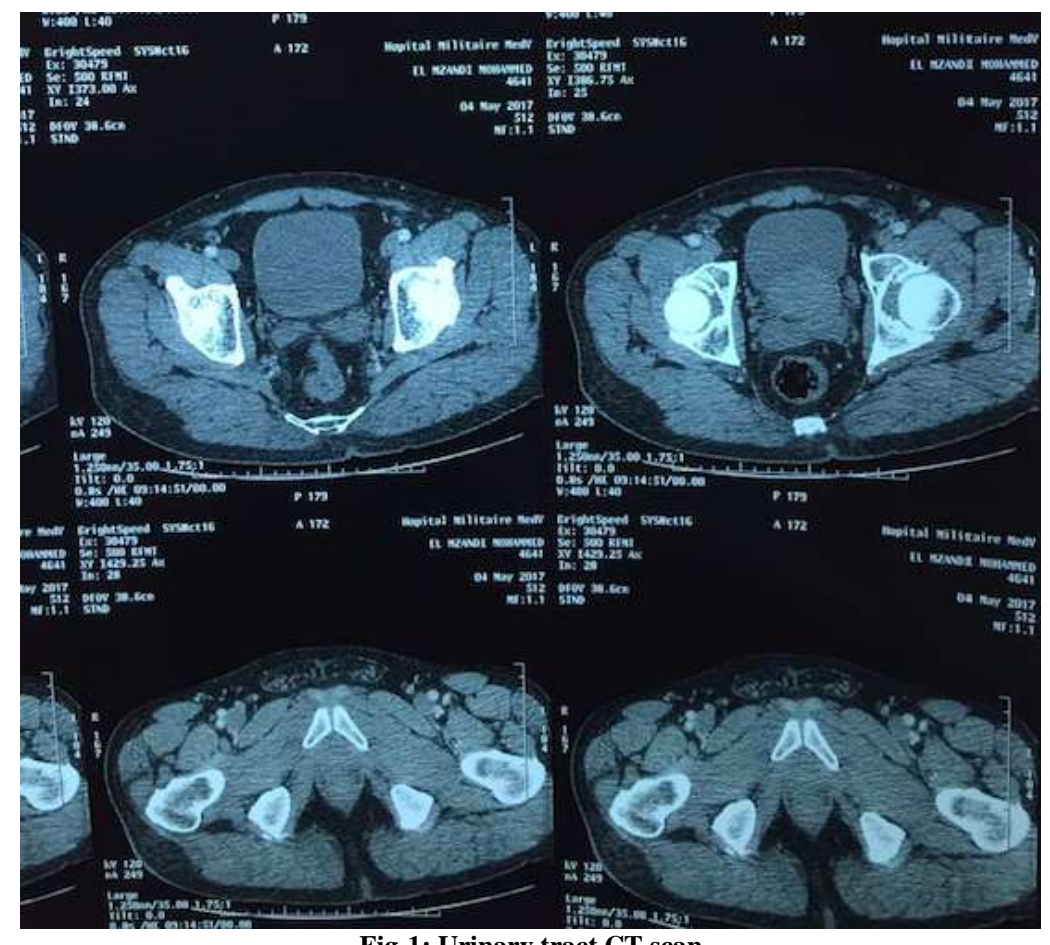

Fig-1: Urinary tract CT scan 


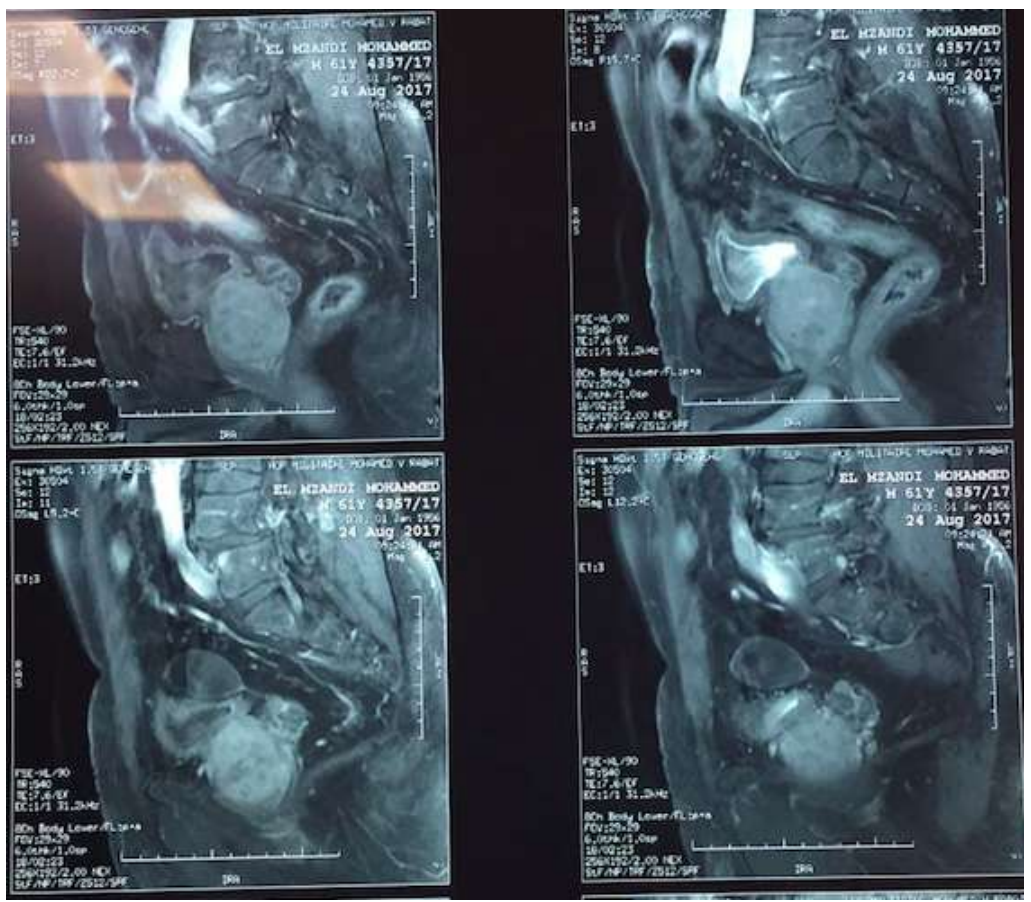

Fig-2: Pelvic MRI showing the bladder wall hydatic cyst

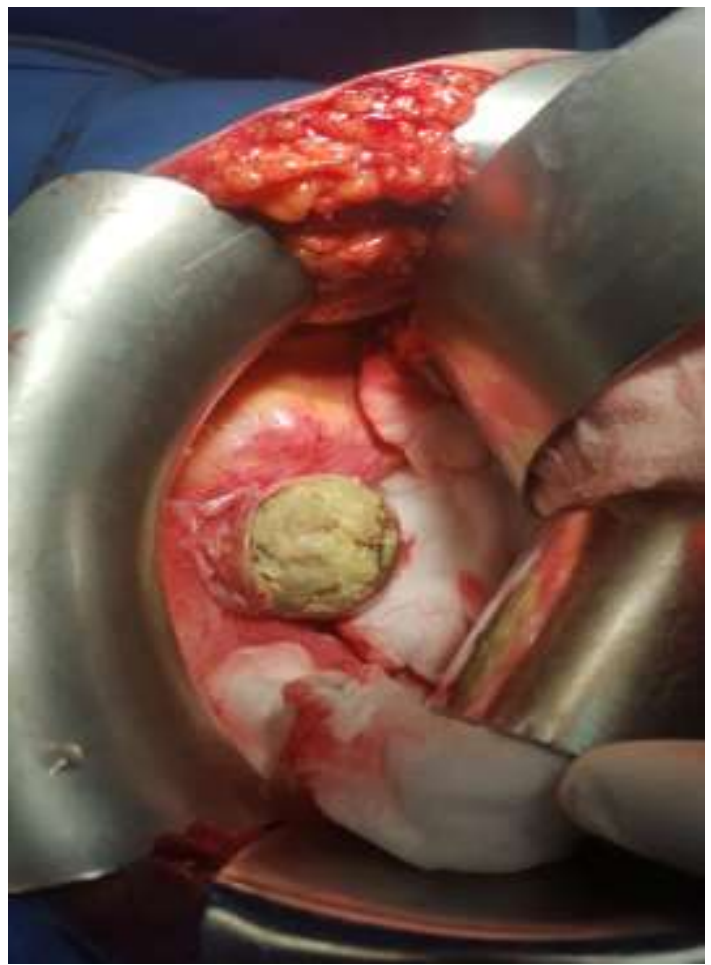

Fig-3: Bladder wall Hydatic cyst image during operation

\section{REFERENCES}

1. Gharbi HA, Hassine W, Brauner MW. Ultrasond examination of hydatic liver. Radiology. 1981,139:459-63.

2. Atan A, Basar MM, Akalin ZA. Case of hydatid disease with unusual localisations. Scand J Urol Nephrol. 1998,32:362-4.

3. Angulo JC, Escribano J, Diego A, SanchezChapado M. Isolated retrovesical and extrarenal retroperitoneal hydatidosis: clinical study of 10 cases and literature review. J Urol. 1998, 159:7682.

4. Rizza G, Bartolotta G, Vacirca F, Rasa G, Pecorella G, Fusto A. Retrovesical hydatid cyst fistulized into the bladder, associated with multiple hepatic and epiploic hydatidosis. Chir Ital 1980, 32:1394-414.

5. Rao MS, Sharma SK, Rajendran L. Non-operative management of a pelvic hydatid cyst communicating with the bladder. J Urol. 1979, 121:245-7.

6. Shetty SD, Al-Saigh A, Ibrahim AI, Patil KP, Bhattachan CL. Management of hydatid cysts of the urinary tract. Br J Urol. 1992, 70:258-61.

7. Von Sinner WN. New diagnostic signs in hydatic disease, radiography, ultrasound, CT and MRI correlated to pathology. Eur J Radiol. 1991,12:1509. 